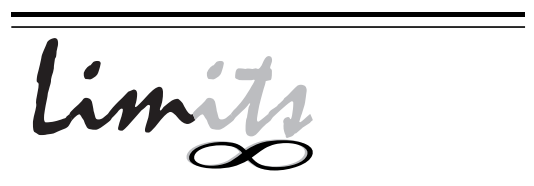

J. Math. and Its Appl.

ISSN : $1829-605 \mathrm{X}$

Vol. 4, No. 2, November 2007, 45-51

\title{
KONSTRUKSI RUANG 2-NORM SEBAGAI LUASAN YANG DIRENTANG OLEH DUA VEKTOR
}

\author{
Sadjidon $^{1}$, H. Gunawan ${ }^{2}$ \\ ${ }^{1}$ Jurusan Matematika, ${ }^{2}$ Departemen Matematika \\ ${ }^{1}$ Institut Teknologi Sepuluh Nopember, Surabaya \\ ${ }^{2}$ Institut Teknologi Bandung, Bandung \\ ${ }^{2}$ hgunawan@dns.math.itb.ac.id
}

\begin{abstract}
Abstrak
Pada paper ini akan dikaji tentang pengkonstruksian ruang 2-norm yang didasari oleh sifat-sifat orthogonalitas dari dua vektor sehingga diperoleh pendefinisikan ruang 2-norm, khususnya untuk ruang $\ell^{2}$.
\end{abstract} Katakunci: Ruang $\ell^{2}$, orthogonalitas, ruang 2-norm.

\section{Pendahuluan}

Ruang $\ell^{2}$ yang dilengkapi dengan inner product $\langle x, y\rangle=\sum_{j} x_{j} y_{j}$, merupakan ruang inner product. Begitu juga ruang $\ell^{2}$ yang dilengkapi dengan norma $\|x\|=\left\{\sum_{k=1}^{\infty}\left|x_{k}\right|^{2}\right\}^{\frac{1}{2}}$ merupakan ruang Banach. Selanjutnya dual dari ruang $\ell^{2}$ yaitu himpunan dari semua fungsional linier kontinu pada ruang $\ell^{2}$ yang dinotasikan dengan $\left(\ell^{2}\right)^{*}$ adalah ruang $\ell^{2}$ juga. Jika $f \in\left(\ell^{2}\right)^{*}$, 
maka $f \in \ell^{2}$ dan dapat diinterpretasikan untuk $f(x)=\sum_{j} x_{j} z_{j}=\langle x, z\rangle$, dengan $x \in \ell^{2}, z \in\left(\ell^{2}\right)^{*}=\ell^{2}$.

Sekarang pandang $S$ himpunan semua barisan bilangan real dan merupakan ruang vektor atas field $R$. Setiap subruang vektor $S$ juga merupakan ruang barisan. Untuk $X$ subruang $S$ didefinisikan suatu fungsi bernilai real $\|\bullet, \ldots, \bullet\|$ pada $X^{n}$ yang memenuhi sifat-sifat sebagai berikut :

1. $\left\|x_{1}, x_{2}, \ldots, x_{n}\right\|=0$, jika dan hanya jika $x_{1}, x_{2}, \ldots, x_{n}$ dependen linier

2. $\left\|x_{1}, x_{2}, \ldots, x_{n}\right\|$ invarian terhadap permutasi

3. $\left\|x_{1}, x_{2}, \ldots, \alpha x_{n}\right\|=|\alpha|\left\|x_{1}, x_{2}, \ldots, x_{n}\right\|$ untuk setiap $\alpha \in R$

4. $\left\|x_{1}, x_{2}, \ldots, x_{n-1}, y+z\right\| \leq\left\|x_{1}, x_{2}, \ldots, x_{n-1}, y\right\|+\left\|x_{1}, x_{2}, \ldots, x_{n-1}, z\right\|$

disebut $n$-norma pada $X$ dan pasangan $(X,\|\bullet, \ldots, \bullet\|)$ disebut ruang $n$ norma.

Pada [2], [3] telah dikonstruksi dan dijabarkan tentang 2-norma, yang disebut sebagai 2-norma standar, selanjutnya dengan memperhatikan sifatsifat orthogonalitas dari [1], [4], maka dikonstruksi 2-norma sehingga diperoleh pendefinisian ruang 2-norm.

\section{Ruang $\ell^{2}$ dan $n$-norma Standarnya}

Sebelum menjabarkan n-norma dijelaskan untuk 2-norma pada ruang $\ell^{2}$ yang diberikan sebagai berikut :

$$
\|x, y\|=\operatorname{Sup}\left\{\left|\begin{array}{cc}
\langle x, z\rangle & \langle y, z\rangle \\
\langle x, w\rangle & \langle y, w\rangle
\end{array}\right|: z, w \in \ell^{2},\|z\|,\|w\| \leq 1\right\} .
$$

Selanjutnya dengan ketaksamaan Cauchy-Schwarz diperoleh

$$
\left|\begin{array}{cc}
\langle x, z\rangle & \langle y, z\rangle \\
\langle x, w\rangle & \langle y, w\rangle
\end{array}\right| \leq\left|\begin{array}{cc}
\langle x, x\rangle & \langle x, y\rangle \\
\langle y, x\rangle & \langle y, y\rangle
\end{array}\right|^{\frac{1}{2}}\left|\begin{array}{cc}
\langle z, z\rangle & \langle z, w\rangle \\
\langle w, z\rangle & \langle w, w\rangle
\end{array}\right|^{\frac{1}{2}} \leq\left|\begin{array}{cc}
\langle x, x\rangle & \langle x, y\rangle \\
\langle y, x\rangle & \langle y, y\rangle
\end{array}\right|^{\frac{1}{2}} .
$$


Hasil ini menunjukkan bahwa $\left|\begin{array}{cc}\langle x, x\rangle & \langle x, y\rangle \\ \langle y, x\rangle & \langle y, y\rangle\end{array}\right|^{\frac{1}{2}}$ merupakan batas atas dari himpunan $\left\{\left|\begin{array}{cc}\langle x, z\rangle & \langle y, z\rangle \\ \langle x, w\rangle & \langle y, w\rangle\end{array}\right|: z, w \in \ell^{2},\|z\|,\|w\| \leq 1\right\}$ dan ini berarti bahwa :

$$
\operatorname{Sup}\left\{\left|\begin{array}{cc}
\langle x, z\rangle & \langle y, z\rangle \\
\langle x, w\rangle & \langle y, w\rangle
\end{array}\right|: z, w \in \ell^{2},\|z\|,\|w\| \leq 1\right\} \leq\left|\begin{array}{cc}
\langle x, x\rangle & \langle x, y\rangle \\
\langle y, x\rangle & \langle y, y\rangle
\end{array}\right|^{\frac{1}{2}}
$$

Selanjutnya untuk $z=\frac{x}{\|x\|} ; w=\frac{y-\alpha x}{\|y-\alpha x\|}=\frac{y^{\prime}}{\left\|y^{\prime}\right\|}$ dengan $z$ dan $y^{\prime}$ orthogonal, juga memenuhi $\|z\|,\|w\| \leq 1$, maka diperoleh

$$
\begin{aligned}
\left|\begin{array}{cc}
\langle x, z\rangle & \langle y, z\rangle \\
\langle x, w\rangle & \langle y, w\rangle
\end{array}\right| & =\left|\begin{array}{cc}
\left\langle x, \frac{x}{\|x\|}\right\rangle & \left\langle y, \frac{x}{\|x\|}\right\rangle \\
\left\langle x, \frac{y^{\prime}}{\left\|y^{\prime}\right\|}\right\rangle & \left\langle y, \frac{y^{\prime}}{\left\|y^{\prime}\right\|}\right\rangle
\end{array}\right| \\
& =\frac{\left|\begin{array}{cc}
\langle x, x\rangle & \langle y, x\rangle \\
\left\langle x, y^{\prime}\right\rangle & \left\langle y, y^{\prime}\right\rangle
\end{array}\right|}{\|x\|\left\|y^{\prime}\right\|} \\
& =\frac{\left|\begin{array}{cc}
\langle x, x\rangle & \langle y, x\rangle \\
\langle x, y\rangle & \langle y, y\rangle
\end{array}\right|}{\|x\|\left\|y^{\prime}\right\|} .
\end{aligned}
$$

dengan menggunakan sifat-sifat determinan dan sifat-sifat inner product diperoleh juga bahwa

$$
\left|\begin{array}{cc}
\langle x, x\rangle & \langle y, x\rangle \\
\langle x, y\rangle & \langle y, y\rangle
\end{array}\right|^{\frac{1}{2}}=\left|\begin{array}{cc}
\langle x, x\rangle & \langle y, x\rangle \\
\left\langle x, y^{\prime}\right\rangle & \left\langle y, y^{\prime}\right\rangle
\end{array}\right|^{\frac{1}{2}}=\left|\begin{array}{cc}
\langle x, x\rangle & \left\langle y^{\prime}, x\right\rangle \\
\left\langle x, y^{\prime}\right\rangle & \left\langle y^{\prime}, y^{\prime}\right\rangle
\end{array}\right|^{\frac{1}{2}}=\|x\|\left\|y^{\prime}\right\|
$$

sehingga

$$
\left|\begin{array}{cc}
\langle x, z\rangle & \langle y, z\rangle \\
\langle x, w\rangle & \langle y, w\rangle
\end{array}\right|=\frac{\|x\|\left\|y^{\prime}\right\|\left|\begin{array}{cc}
\langle x, x\rangle & \langle y, x\rangle \\
\langle y, x\rangle & \langle y, y\rangle
\end{array}\right|^{\frac{1}{2}}}{\|x\|\left\|y^{\prime}\right\|}=\left|\begin{array}{cc}
\langle x, x\rangle & \langle y, x\rangle \\
\langle x, y\rangle & \langle y, y\rangle
\end{array}\right|^{\frac{1}{2}} .
$$

Hasil ini menunjukkan bahwa

$$
\begin{aligned}
\left|\begin{array}{cc}
\langle x, x\rangle & \langle y, x\rangle \\
\langle x, y\rangle & \langle y, y\rangle
\end{array}\right|^{\frac{1}{2}} & =\left|\begin{array}{cc}
\langle x, z\rangle & \langle y, z\rangle \\
\langle x, w\rangle & \langle y, w\rangle
\end{array}\right| \\
& \leq \operatorname{Sup}\left\{\left|\begin{array}{cc}
\langle x, z\rangle & \langle y, z\rangle \\
\langle x, w\rangle & \langle y, w\rangle
\end{array}\right|: z, w \in \ell^{2},\|z\|,\|w\| \leq 1\right\}
\end{aligned}
$$

Dengan demikian dari Persamaan (1) dan Persamaan (2) diperoleh 2-norma pada ruang $\ell^{2}$ adalah

$$
\|x, y\|=\operatorname{Sup}\left\{\left|\begin{array}{cc}
\langle x, z\rangle & \langle y, z\rangle \\
\langle x, w\rangle & \langle y, w\rangle
\end{array}\right|: z, w \in \ell^{2},\|z\|,\|w\| \leq 1\right\}=\left|\begin{array}{cc}
\langle x, x\rangle & \langle x, y\rangle \\
\langle y, x\rangle & \langle y, y\rangle
\end{array}\right|^{\frac{1}{2}}
$$


dan 2-Norma $\|x, y\|$ tidak lain adalah luasan yang direntang oleh vektor-vektor $x$ dan $y$. Selanjutnya akan dijabarkan untuk n-normanya dalam ruang $\ell^{2}$ yang dikenal sebagai ruang inner product dengan inner product $\langle x, y\rangle=\sum_{j} x_{j} y_{j}$, dapat dilengkapi dengan $n$-normanya

$$
\left\|x_{1}, x_{2}, \ldots, x_{n}\right\|=\left|\begin{array}{ccc}
\left\langle x_{1}, x_{1}\right\rangle & \ldots & \left\langle x_{1}, x_{n}\right\rangle \\
. . & . . & . . \\
\left\langle x_{n}, x_{1}\right\rangle & \ldots & \left\langle x_{n}, x_{n}\right\rangle
\end{array}\right|^{\frac{1}{2}}
$$

merupakan ruang $n$-norma standar sehingga ruang $\ell^{2}$ merupakan ruang $n$-norma. Khususnya jika $n=2$, maka 2-norma standar untuk ruang $\ell^{2}$ adalah :

$$
\|x, y\|=\left|\begin{array}{ll}
\langle x, x\rangle & \langle x, y\rangle \\
\langle y, x\rangle & \langle y, y\rangle
\end{array}\right|^{\frac{1}{2}}
$$

Untuk $n$-norma pada ruang $\ell^{p}$ khususnya ruang $\ell^{2}$, penjabarannya dan pengembangannya dalam [2].

Sekarang akan dijabarkan $n$-norma pada ruang $\ell^{2}$ menurut pendefinisian [1] dengan $n$-norma nya sebagai berikut :

$$
\begin{aligned}
& \left\|x_{1}, \ldots, x_{n}\right\| \\
& =\operatorname{Sup}\left\{\left|\begin{array}{ccc}
f_{1}\left(x_{1}\right) & \ldots & f_{1}\left(x_{n}\right) \\
. . & \ldots & . . \\
f_{n}\left(x_{1}\right) & \ldots & f_{n}\left(x_{n}\right)
\end{array}\right|: f_{1}, \ldots, f_{n} \in\left(\ell^{2}\right)^{*}=\ell^{2},\left\|f_{1}\right\|, \ldots,\left\|f_{n}\right\| \leq 1\right\}
\end{aligned}
$$

atau dapat dituliskan

$$
\begin{aligned}
& \left\|x_{1}, \ldots, x_{n}\right\| \\
& =\operatorname{Sup}\left\{\left|\begin{array}{ccc}
\left\langle x_{1}, z_{1}\right\rangle & \ldots & \left\langle x_{1}, z_{n}\right\rangle \\
. . & \ldots & . . \\
\left\langle x_{n}, z_{1}\right\rangle & \ldots & \left\langle x_{n}, z_{n}\right\rangle
\end{array}\right|: z_{1}, \ldots, z_{n} \in \ell^{2},\left\|z_{1}\right\|, \ldots,\left\|z_{n}\right\| \leq 1\right\} .
\end{aligned}
$$

Selanjutnya dengan ketaksamaan Cauchy-Schwarz didapatkan

$$
\begin{aligned}
\left|\begin{array}{ccc}
\left\langle x_{1}, z_{1}\right\rangle & \ldots & \left\langle x_{1}, z_{n}\right\rangle \\
. . & \ldots & . . \\
\left\langle x_{n}, z_{1}\right\rangle & \ldots & \left\langle x_{n}, z_{n}\right\rangle
\end{array}\right| & \leq\left|\begin{array}{ccc}
\left\langle x_{1}, x_{1}\right\rangle & \ldots & \left\langle x_{1}, x_{n}\right\rangle \\
. . & \ldots & . . \\
\left\langle x_{n}, x_{1}\right\rangle & \ldots & \left\langle x_{n}, x_{n}\right\rangle
\end{array}\right|^{\frac{1}{2}}\left|\begin{array}{ccc}
\left\langle z_{1}, z_{1}\right\rangle & \ldots & \left\langle z_{1}, z_{n}\right\rangle \\
. . & \ldots & \ldots \\
\left\langle z_{n}, z_{1}\right\rangle & \ldots & \left\langle z_{n}, z_{n}\right\rangle
\end{array}\right|^{\frac{1}{2}} \\
& \leq\left|\begin{array}{ccc}
\left\langle x_{1}, x_{1}\right\rangle & \ldots & \left\langle x_{1}, x_{n}\right\rangle \\
. . & \ldots & . . \\
\left\langle x_{n}, x_{1}\right\rangle & \ldots & \left\langle x_{n}, x_{n}\right\rangle
\end{array}\right|^{\frac{1}{2}}
\end{aligned}
$$


ini menunjukkan $\left|\begin{array}{ccc}\left\langle x_{1}, x_{1}\right\rangle & \ldots & \left\langle x_{1}, x_{n}\right\rangle \\ . . & \ldots & . . \\ \left\langle x_{n}, x_{1}\right\rangle & \ldots & \left\langle x_{n}, x_{n}\right\rangle\end{array}\right|^{\frac{1}{2}}$ batas atas dari himpunan

$$
\left\{\left|\begin{array}{ccc}
\left\langle x_{1}, z_{1}\right\rangle & \ldots & \left\langle x_{1}, z_{n}\right\rangle \\
. . & \ldots & . . \\
\left\langle x_{n}, z_{1}\right\rangle & \ldots & \left\langle x_{n}, z_{n}\right\rangle
\end{array}\right|: z_{1}, \ldots, z_{n} \in \ell^{2},\left\|z_{1}\right\|, \ldots,\left\|z_{n}\right\| \leq 1\right\}
$$

berarti bahwa

$$
\begin{aligned}
& \left\|x_{1}, \ldots, x_{n}\right\| \\
& =\operatorname{Sup}\left\{\left|\begin{array}{ccc}
f_{1}\left(x_{1}\right) & \ldots & f_{1}\left(x_{n}\right) \\
. . & \ldots & . . \\
f_{n}\left(x_{1}\right) & \ldots & f_{n}\left(x_{n}\right)
\end{array}\right|: f_{1}, \ldots, f_{n} \in\left(\ell^{2}\right)^{*}=\ell^{2},\left\|f_{1}\right\|, \ldots,\left\|f_{n}\right\| \leq 1\right\} \\
& \leq\left|\begin{array}{ccc}
\left\langle x_{1}, x_{1}\right\rangle & \ldots & \left\langle x_{1}, x_{n}\right\rangle \\
. . & \ldots & . . \\
\left\langle x_{n}, x_{1}\right\rangle & \ldots & \left\langle x_{n}, x_{n}\right\rangle
\end{array}\right| .
\end{aligned}
$$

Selanjutnya untuk $\left\{z_{1}, z_{2}, \ldots, z_{n}\right\}$ yang merupakan hasil proses orthonormalisasi Gram-Schmidt terhadap $\left\{x_{1}, x_{2}, \ldots, x_{n}\right\}$, juga memenuhi $\left\|z_{1}\right\|,\left\|z_{2}\right\|, \ldots \ldots,\left\|z_{n}\right\|=1$, maka diperoleh

$$
\left|\begin{array}{ccc}
\left\langle x_{1}, z_{1}\right\rangle & \ldots & \left\langle x_{1}, z_{n}\right\rangle \\
. . & \ldots & . . \\
\left\langle x_{n}, z_{1}\right\rangle & \ldots & \left\langle x_{n}, z_{n}\right\rangle
\end{array}\right|=\left|\begin{array}{ccc}
\left\langle x_{1}, x_{1}\right\rangle & \ldots & \left\langle x_{1}, x_{n}\right\rangle \\
. . & \ldots & . . \\
\left\langle x_{n}, x_{1}\right\rangle & \ldots & \left\langle x_{n}, x_{n}\right\rangle
\end{array}\right|^{\frac{1}{2}}
$$

Hasil ini menunjukkan bahwa

$$
\begin{aligned}
& \left|\begin{array}{ccc}
\left\langle x_{1}, x_{1}\right\rangle & \ldots & \left\langle x_{1}, x_{n}\right\rangle \\
. . & \ldots & . . \\
\left\langle x_{n}, x_{1}\right\rangle & \ldots & \left\langle x_{n}, x_{n}\right\rangle
\end{array}\right|^{\frac{1}{2}}=\left|\begin{array}{ccc}
\left\langle x_{1}, z_{1}\right\rangle & \ldots & \left\langle x_{1}, z_{n}\right\rangle \\
. . & \ldots & . . \\
\left\langle x_{n}, z_{1}\right\rangle & \ldots & \left\langle z_{n}, z_{n}\right\rangle
\end{array}\right| \\
& \leq \operatorname{Sup}\left\{\left|\begin{array}{ccc}
\left\langle x_{1}, z_{1}\right\rangle & \ldots & \left\langle x_{1}, z_{n}\right\rangle \\
. . & \ldots & . . \\
\left\langle x_{n}, z_{1}\right\rangle & \ldots & \left\langle x_{n}, z_{n}\right\rangle
\end{array}\right|: z_{1}, \ldots, z_{n} \in \ell^{2},\left\|z_{1}\right\|, \ldots,\left\|z_{n}\right\| \leq 1\right\}
\end{aligned}
$$

Dengan demikian dapat diperoleh

$$
\begin{array}{r}
\left\|x_{1}, \ldots, x_{n}\right\|=\operatorname{Sup}\left\{\left|\begin{array}{ccc}
\left\langle x_{1}, z_{1}\right\rangle & \ldots & \left\langle x_{1}, z_{n}\right\rangle \\
. . & \ldots & . . \\
\left\langle x_{n}, z_{1}\right\rangle & \ldots & \left\langle x_{n}, z_{n}\right\rangle
\end{array}\right|: z_{1}, \ldots, z_{n} \in \ell^{2},\right. \\
\left.\left\|z_{1}\right\|, \ldots,\left\|z_{n}\right\| \leq 1\right\}=\left|\begin{array}{ccc}
\left\langle x_{1}, x_{1}\right\rangle & \ldots & \left\langle x_{1}, x_{n}\right\rangle \\
. . & \ldots & . . \\
\left\langle x_{n}, x_{1}\right\rangle & \ldots & \left\langle x_{n}, x_{n}\right\rangle
\end{array}\right|^{\frac{1}{2}}
\end{array}
$$


Sekarang diberikan fungsional linier pada $\ell^{2} \times \ell^{2}$ yang diberikan

$$
f(u)=\sum_{k=1}^{\infty}\left(x_{k}^{1}+x_{k}^{2}\right) w_{k}=\left\langle x_{1}, w\right\rangle+\left\langle x_{2}, w\right\rangle
$$

dengan $u=\left(x_{1}, x_{2}\right) \in \ell^{2} \times \ell^{2}$ dan $w \in\left(\ell^{2}\right)^{*}=\ell^{2}$ dan $\|u\|=\left\|x_{1}\right\|+\left\|x_{2}\right\|$ Maka 2-Norma pada ruang $\ell^{2} \times \ell^{2}$ didefinisikan menurut [1] dapat disajikan dengan

$$
\|u, v\|=\operatorname{Sup}\left\{\left|\begin{array}{cc}
f(u) & f(v) \\
f(v) & g(v)
\end{array}\right|, f, g \in\left(\ell^{2}\right)^{*}=\ell^{2},\|f\|,\|g\| \leq 1\right\}
$$

dengan $u=\left(x_{1}, x_{2}\right) \quad, \quad v=\left(y_{1}, y_{2}\right)$. Sehingga dapat dituliskan dengan

$$
\|u, v\|=\operatorname{Sup}\left\{\left|\begin{array}{cc}
\left\langle x_{1}+x_{2}, w\right\rangle & \left\langle y_{1}+y_{2}, w\right\rangle \\
\left\langle x_{1}+x_{2}, z\right\rangle & \left\langle\mathrm{y}_{1}+y_{2}, z\right\rangle
\end{array}\right|, w, z \in\left(\ell^{2}\right)^{*}=\ell^{2},\|w\|,\|z\| \leq 1\right\} .
$$

Selanjutnya dengan ketaksamaan Cauchy-Schwarz diperoleh

$$
\left|\begin{array}{cc}
\left\langle x_{1}+x_{2}, w\right\rangle & \left\langle y_{1}+y_{2}, w\right\rangle \\
\left\langle x_{1}+x_{2}, z\right\rangle & \left\langle\mathrm{y}_{1}+y_{2}, z\right\rangle
\end{array}\right| \leq\left\|x_{1}, y_{1}\right\|+\left\|x_{1}, y_{2}\right\|+\left\|x_{2}, y_{1}\right\|+\left\|x_{2}, y_{2}\right\|=\|u\|\|v\|
$$

Hasil ini menunjukkan bahwa $\|u\|\|v\|$ batas atas dari himpunan

$$
\left\{\left|\begin{array}{cc}
\left\langle x_{1}+x_{2}, w\right\rangle & \left\langle y_{1}+y_{2}, w\right\rangle \\
\left\langle x_{1}+x_{2}, z\right\rangle & \left\langle\mathrm{y}_{1}+y_{2}, z\right\rangle
\end{array}\right|, w, z \in\left(\ell^{2}\right)^{*}=\ell^{2},\|w\|,\|z\| \leq 1\right\}
$$

dengan demikian

$$
\left.\begin{array}{l}
\|u, v\| \\
=\operatorname{Sup}\left\{\left|\begin{array}{cc}
\left\langle x_{1}+x_{2}, w\right\rangle & \left\langle y_{1}+y_{2}, w\right\rangle \\
\left\langle x_{1}+x_{2}, z\right\rangle & \left\langle\mathrm{y}_{1}+y_{2}, z\right\rangle
\end{array}\right|, w, z \in\left(\ell^{2}\right)^{*}=\ell^{2},\|w\|,\|z\| \leq 1\right.
\end{array}\right\}
$$

Dari hasil yang dijabarkan diatas tentang 2-norma pada $\ell^{2} \times \ell^{2}$ dapat dilanjutkan 2-norma untuk ruang $\left(\ell^{2}\right)^{n}=\ell^{2} \times \ldots . . \times \ell^{2}$. Untuk itu dalam $\ell^{2} \times \ell^{2}$ apakah suatu luasan yang dibentang dari jumlahan vektor yaitu $\left(x_{1}+x_{2}\right)$ dan $\left(y_{1}+y_{2}\right)$, begitu juga dalam $\left(\ell^{2}\right)^{n}=\ell^{2} \times \ldots . . \times \ell^{2}$.apakah luasan yang dibentang oleh jumlahan dari vektor.

\section{Pustaka}

[1] C.R. Diminnie, A New Orthogonality Relation for Normed Linear Spaces, Math.Nachr.114 (197-203), 1983. 
[2] H. Gunawan dan M. Mashadi, On n-normed spaces, Int.J.Math.Sci, (to appear).

[3] H. Gunawan, The space of p-Summable sequences and its natural n-Norm, Bull.Austral.Math.Soc, Vol.64(137-147), 2001.

[4] J.R Partington, Orthogonality in Normed Spaces, Bull.Austral.Math.Soc.33 (449-455), 1986.

[5] Kreyszig, Introductory Fuctional Analysis with Applications, John Wiley and Son. Inc, 1978. 